\title{
Numerical Prediction of Resistance of Planning Vessel with RANS Method
}

\author{
YuCheng Wang \\ China Ship Development and Design Center, Wu Han, 430064i, China
}

yuchengw1987@163.com

\section{Keywords: Planning Vessel; Resistance; CFD; RANS; Ship Motion Equation}

\begin{abstract}
In order to get the accurate resistance of planning vessel at high speed, we should consider the hull gesture variation during voyage. Based on CFD, coupling solves RANS and ship motion equation to capture the ship navigation attitude and resistance. The numerical calculation gets the resistance and navigation attitude of the planning vessel and compared with the corresponding experimental date, the result shows its accuracy can meet the requirement of engineering application. The ship designers can use the CFD result to design the shape of ship hull.
\end{abstract}

\section{Introduction}

Because of their excellent performance with respect to speed, resistance and transversal stability, the demand for high-speed vessels has strongly increased for both commerical and military during the last decades; expecially, the high speed catamaran configurations are very attractive. In the contrast to the conventional displacment ship which is supported primarily by the hydrostatic buoyancy force, the high speed catamaran has a substantial portion of its weight supported by the hydrodynamic lift[1]. However, requirements for planning vessels in the design process need the use of larger width of boat and draft based on the actual demand, which results in large displacement length coefficient of the hull. One of the key technologies for planning boat hull design is the accurate prediction of its hydrodynamic properties. The resistance prediction of real ship has been the close attention object of shipbuilding world. Ship resistance prediction method is mainly theoretical research, the ship pool test and CFD numerical calculation. When the ship sailed planning, its force becomes complicated. The change of dynamic pressure and viscous forces brings the attitude motion of hull, the vertical force and pitching torque of hull will change, results in the rise and longitudinal motion of hull. Thus, the attitude of ship at planning is different from the one at the hydrostatic floating state. As it is more effectively applied in today's modern military and pleasure craft, and offshore industry supply vessels, there is increasing demand to further develop the catamaran's planning and seaking characteristics [2].The high speed catamaran faces dynamic instability problems in both transerverse and vertical planes, duing the complicated nature of the hydrodynamic forces [3][4][5].The research on the study of the vertical-plane motion of high speed cafts became a very active and fruiful field which can be traced back to the early twentieth century; in particular ,during 1960s-1990s[6-11].These earlier works focuesd mainly on the effects of design parameters, such as the forward speed, the location of the center of gravity, load and other geometric parameters of the high speed vessels, on the characteristical motion of vessels.

The lift and longitudinal motion of hull at low speed in general will not be much. The change of ship resistance value which can't be considered in the hull resistance forecasting process is not evident. When the ship sails at planning segment, the vertical and lift motion of hull will have been significantly changed. Therefore, when study the rapidity of high-speed planning vessel, we need consider the impact of attitude change on its resistance during voyage.

Su[12] presented a new CFD method which can calculate hydrodynamic forces of high speed planing vessel. The numerical method is based on RANS equations. They applied the VOF method and six-degrees-of-freedom equation. Dynamic stability is important for planning vessels. Porpoising is one of the most concerned instability problems for this type of high speed vessel. Sun[13] present the $2 \mathrm{D}+\mathrm{t}$ approach to simulate the free motions of a planning vessel under an initial disturbance in calm water. Yao[14] studied the effects of dead rise on the resistance performance of 
deep-vee planning craft by model test, they found that a suitable dead rise angle can reduce the resistance. Wang[15] studied the accuracy of the calculation of planning crafts hydrodynamic forces in the steady sailing attitude based on the RANS VOF solver. In order to get the proper mesh generation for the planning vessel flow field simulation, $\mathrm{Ma}[16]$ researched the applicability of the hybrid grids. Model tests were conducted by Sun[17] to verify the ability of a trimaran planning boat sailing at ultra high speeds and to investigate its characteristics of resistance.

\section{Calculation Model}

\section{Governing equations}

By using Fine-Marine software to calculate the planning vessel model, the governing equations for the continuity equation and Reynolds stress averaged equations are [18]:

$$
\begin{aligned}
& \frac{\partial \rho}{\partial t}+\frac{\partial}{\partial x_{i}}\left(\rho u_{i}\right)=0 \\
& \frac{\partial}{\partial t}\left(\rho u_{i}\right)+\frac{\partial}{\partial x_{j}}\left(\rho u_{i} u_{j}\right)=-\frac{\partial p}{\partial x_{i}}+\frac{\partial}{\partial x_{j}}\left[\mu\left(\frac{\partial u_{i}}{\partial x_{j}}+\frac{\partial u_{j}}{\partial x_{i}}-\frac{2}{3} \delta_{i j} \frac{\partial u_{i}}{\partial x_{i}}\right)\right]+\frac{\partial}{\partial x_{j}}\left(-\rho \overline{u_{i}^{\prime} u_{j}^{\prime}}\right)
\end{aligned}
$$

Where, $u_{i}$ and $u_{j}$ are the time average amount of rate, $u_{i}^{\prime}$ and $u_{j}^{\prime}$ are the quantity of velocity fluctuations. And compared to the instantaneous NS equations, given the role of turbulence, an extra entryp $\overline{u_{i}^{\prime} u_{j}^{\prime}}$ is Reynolds stresses. The above equation is not closed, in order to achieve a closed equation turbulence model to solve by introducing the Reynolds stress term, achieve fluid control equations can be solved.

\section{Turbulence Model}

Compared to other turbulence models, SST turbulence model which is a model based on the model of the evolution of the developed K-Omega model considers the computational precision and convergence in the process of computation [18-19]. It is set in the near-wall region of viscous flow simulation model to simulate the reliability and accuracy advantages model fully developed turbulence model in the region. And equations of the model are as follows:

$$
\begin{aligned}
& (\rho \kappa)+\frac{\partial}{\partial x_{i}}\left(\rho \kappa u_{i}\right)=\frac{\partial}{\partial x_{j}}\left(\Gamma_{\kappa} \frac{\partial \kappa}{\partial x_{j}}\right)+G_{\kappa}-Y_{\kappa} \\
& \frac{\partial}{\partial t}(\rho \omega)+\frac{\partial}{\partial x_{i}}\left(\rho \omega u_{i}\right)=\frac{\partial}{\partial x_{j}}\left(\Gamma_{\omega} \frac{\partial \omega}{\partial x_{j}}\right)+G_{\omega}-Y_{\omega}+D_{\omega}
\end{aligned}
$$

In the formula, $\Gamma_{\kappa}$ and $\Gamma_{\omega}$ are the diffusion coefficient, $G_{\kappa}$ and $G_{\omega}$ are the turbulence generation items, $Y_{\kappa}$ and $Y_{\omega}$ are the turbulent dissipation terms, $D_{\omega}$ is the diffusion term.

\section{Equations of motion and Calculation Process}

In the process of numerical calculation, the hull movement as a rigid body motion, by using momentum theorem and moment of momentum theorem, we obtained motion equations of the hull six degrees of freedom [16]. Wherein, the movement direction of hull is in the $\mathrm{x}$-axis positive direction, the starboard of hull is in the y-axis positive direction, the anti-direction of gravity is in the z-axis positive direction. In this paper, we consider the impact of hull direct, heave and pitch movement in three directions on resistance at planning during the voyage.

If you still use the constrained model which does not consider attitude changes of hull to predict the resistance of hull, you will find that there is difference between numerical calculations and experimental values. When the Froude number becomes larger, the gap is more obvious. By using the new calculation method which coupled motion equations of hull and RANS, we timely forecast the force of hull. The hull attitude is adjusting according to the force, until it is in dynamic equilibrium. Thus you can accurately predict the stabilized resistance and attitude of planning vessel motion.

\section{Computation Grid}

According to the specific type values of each cross section, by using modeling software to generate three dimensional model of planning vessel, which is shown in Fig.1. In this paper, in 
order to compare the calculation results with the corresponding experimental data, we change the scale of real ship in which its reduced scale is 5 with the same scale as ship test model.

Meshing is one of the most critical parts of the numerical simulation. The quality of the grid directly affects the accuracy of calculation results and convergence time. In this paper, by using the pre-processing Hexpress module in Fine-Marine software, we obtain calculation mesh. The mesh near the surface of hull is suitably refined and projected to the hull surface, thereby body-fitted mesh is forming. At the same time, by splitting the first layer mesh near the hull surface, anisotropic mesh of high quality on boundary layer near the hull surface is generated which is used to accurately capture flow information around the hull. Computational grid is shown in the Fig.1, the overall number of cells is about 425,220. The mesh elements are almost close to rectangular. Wherein, the angle of about 38million grid is up to 80 degrees, the minimum angle is 22.28 degrees.

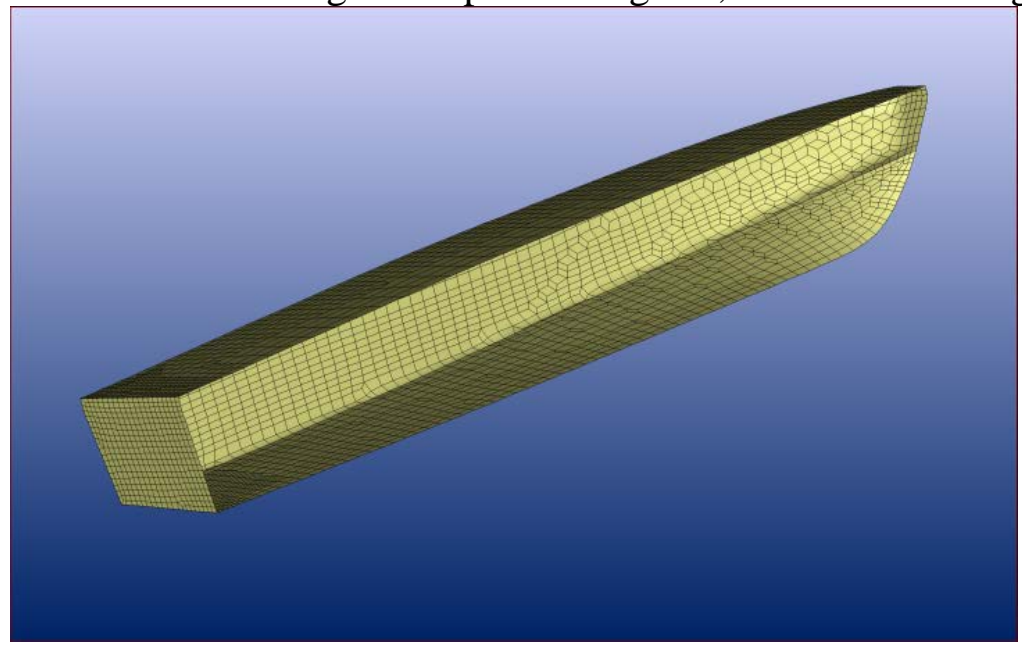

Fig.1 Computational mesh on hull surface

In the numerical calculation process, in order to consider the impact of changes in the hull attitude, the dynamic mesh is used to simulate complex flow field. Grid fluctuates with the movement of hull, its specific move characteristics depends on the hull motion and grid deformation. Mesh deformation can be divided into rigid deformation and weighted deformation. Rigid deformation means that grid moves with the hull, there is no change in the grid shape and relative position between nodes. Weighted deformation means on basis that the total number of grid is same, the solver can adaptively change grid depending on the hull movement of position, the grid can occur pulled or twisted. In this paper, we mainly consider the impact of heave and pitch motion on the resistance of planning vessel. Therefore, the grids are weighted in heave and pitch motion direction, are rigid in other four directions. In the calculation of hull movement coupled with direct flight, heave and pitch, by using the dynamic mesh with weighted deformation and rigid deformation, numerical divergence problem can be effectively avoided which is caused by the deformation of the fine mesh near hull body or distortion.

\section{Numerical Results}

\section{Resistance}

Although the numerical method used by Wang can predict the resistance of high speed planning vessel. Comparing the calculation results with experimental data, the gap between them will have been becoming lager as the Froude number increases. Due to the higher aspect ratio of planning vessel, when the Froude number is greater than 0.8, the hull is affected by the hydrodynamic force, which becomes planning state from displacement state, the proportion of wave making resistance will become smaller, the thin ship theory is no longer applicable. When the towing speed of ship model exceeds $6.0 \mathrm{~m} / \mathrm{s}$, the resistance becomes small, yet the numerical calculation does not produce this phenomenon. The depth of pool is only $5.0 \mathrm{~m}$, while the ship model towing speed has reached $8.5 \mathrm{~m} / \mathrm{s}$, the Froude number of depth has reached 1.2, so that there will be shallow water effects. 


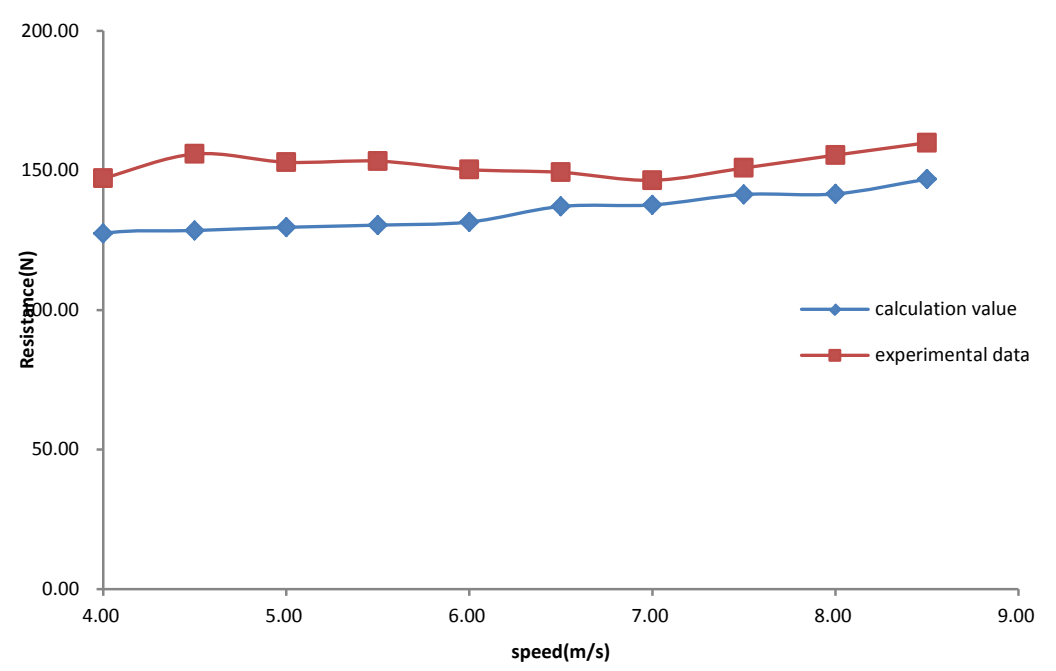

Fig.2 Resistance comparison of model test and numerical calculation

In this paper, by solving the viscous RANS and hull equations of motion to compute the real time power, the attitude for hull adjusted based on the force until it is in dynamic equilibrium. Thus, we can forecast the hull attitude and resistance at particular speed of motion. In Fine-Marine software, calculations are performed using the way of time step to solve for steady or unsteady multiphase flow. Should be expected the steady flow, you can use the steady state to calculate. If expected the transient solution, you must use the transient calculations. In this paper, we mainly forecast the resistance and attitude at stabilized speed of ship motion by using numerical calculation. The final state is stable state, the time step of way is used in the numerical calculation which is set as a constant calculation. Numerical simulation is set steady, as the speed of hull increases, its resistance increases. But when the speed is steady, its resistance value is constant.

\section{Heave motion}

In order to study the effect of hull attitude change on its resistance, we used the dynamic models which account for the hull attitude to forecast the resistance in the numerical calculation process. In the instantaneous of numerical calculation starting, the velocity and pressure fields around the hull will change dramatically, at the same time the force on hull surface will variety with large fluctuations, the hull attitude is adjusted based on the force. After some time, the hull is in dynamic equilibrium, the center of gravity position and pitch angle of hull are stable. Fig. 3 shows the stable heave motion of the center of gravity at different speeds.

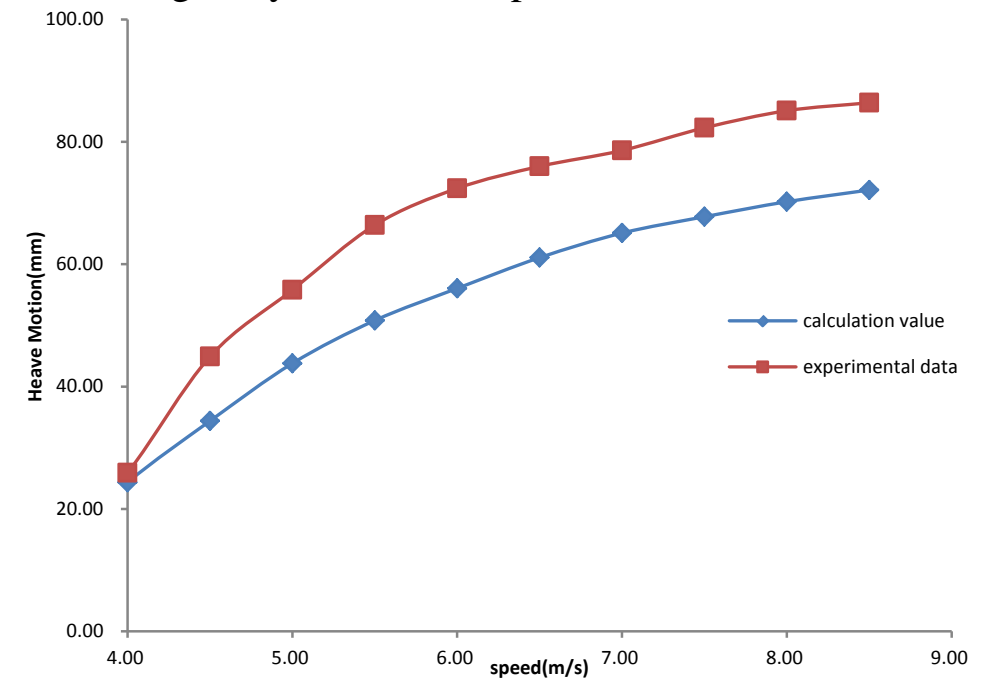

Fig.3 Heave motion comparison of model test and numerical calculation

The data in Fig. 3 shows that the longitudinal displacement hull's center of gravity will continue to move backward, as the speed of hull increases. Although there has been an error between the experimental data and calculated values, yet they remained in a stable range. And there is a consistent trend between experimental data and calculated values. 


\section{Longitudinal angle}

By scaling the planning boat which the reduced scale radio is 5, and carried out the model tests in China Special Vehicle Research Institute, we obtained the pitch angle at different speed. The comparison of the model test data with numerical results is shown in Fig.4.

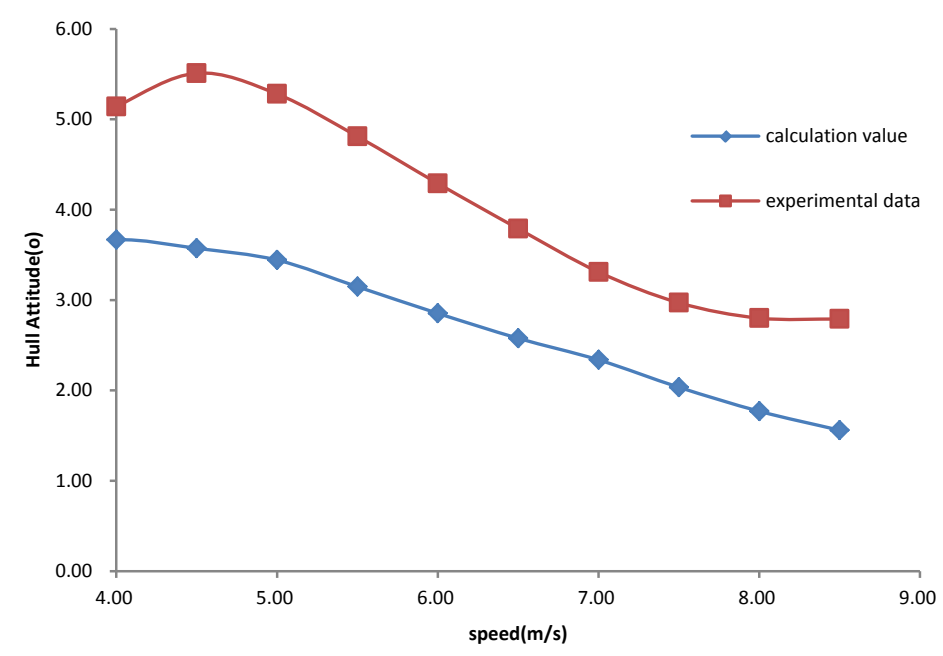

Fig.4 Pitch motion comparison of model test and numerical calculation

As shown in Fig.4, there is a consistent changing trend of hull attitude between the numerical calculation and experiment at the stabilized speed. Whether the test results or numerical results indicate that the attitude of hull is not changing but stabilized at a particular value segment along with the speed increasing, when the speed reaches $4.14 \mathrm{~m} / \mathrm{s}$. When the speed is less than $4.14 \mathrm{~m} / \mathrm{s}$, the stable value of pitch angle will grow up with the increase of speed As shown in Fig.3 and Fig.4, the values of hull heave motion and pitch angle obtained by the numerical simulation are quite different from the experimental ones. In the calculation process, by giving a strong movement in the direction of hull flight and releasing the hull trim and heave motion, we got the point of force which is located at the center of gravity of the hull. But in the experiment, the traction force is in the direction of extended line of propeller shaft. Since the forced movement is not given on the same straight line, there is additional torque in the numerical calculation which is the reason that the errors of hull heave and pitch motion between calculation results and experimental data are larger than one of resistance.

\section{Free surface}

In this section, simulating results of the free surface over different speed is presented. Free surface at different speed is illustrated in Fig.5. Wherein (a) (d) respectively the wave-making cloud of $5.5 \mathrm{~m} / \mathrm{s}, 6.5 \mathrm{~m} / \mathrm{s}, 7.5 \mathrm{~m} / \mathrm{s}, 8.5 \mathrm{~m} / \mathrm{s}$ 。 The results show that the change in the total resistance is not large with the increase in speed, but the tail bow wave will rise higher.
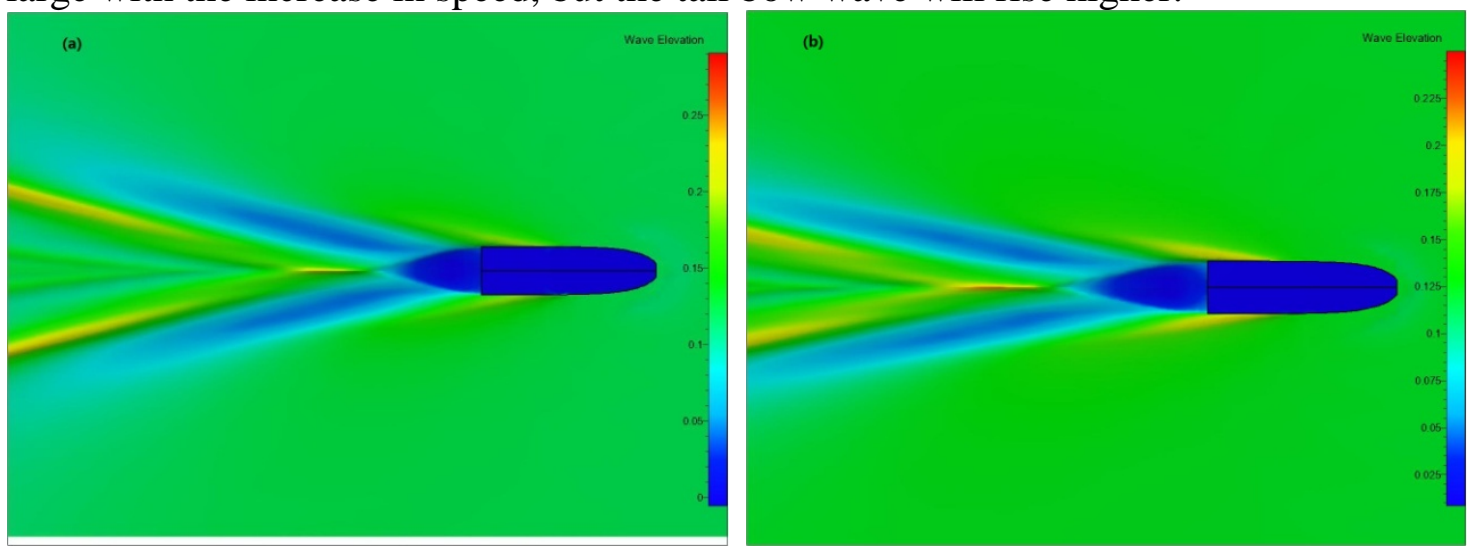

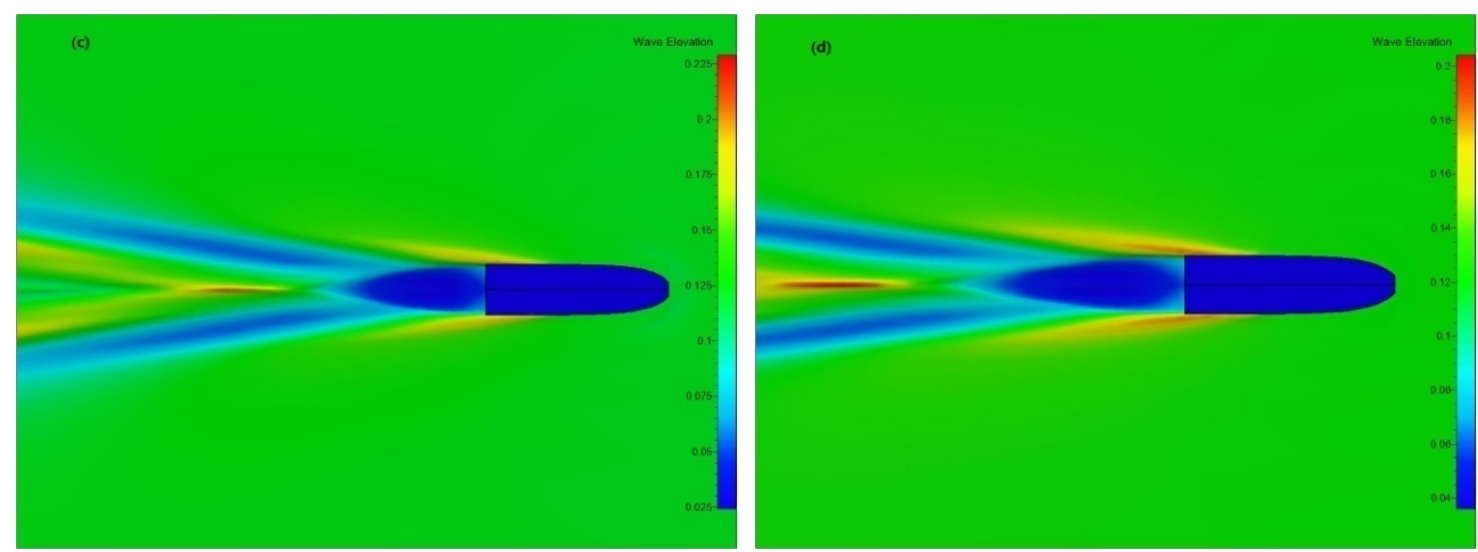

Fig.5. free surface of planning vessel at different speed

\section{Conclusion}

Based on two phase flow theory and dynamic mesh, by solving the equations of hull motion and viscous RANS, we obtained hull forces and accuracy free surface. The hull attitude is adjusting according to the force, until it is in dynamic equilibrium. Thus you can accurately predict the stabilized resistance and attitude of planning vessel motion. By comparing the numerical results with the corresponding experimental data, we found the accuracy can meet the engineering requirements. Ship design workers can design the hull line of planning vessel according to the numerical results, which has important application value. This method not only can accurately forecast hydrodynamic characteristics of planning craft, but also predict hydrodynamic of real boats or other high-performance vessels. For high-performance vessels in waves of different types of 6 DOF motion prediction will be the focus of future research.

\section{References}

[1]Faltinsen,O.M.(2005)Hydrodynamic of High-Speed Marine Vehicles.Cambridge University Press.

[2]Zhengquan Zhou(2003) A Theory and Analysis of Planing Catamarans in Calm and Rough Water.PhD thesis, University of New Orleans.

[3]D.L.Blount and L.T.Codega(1992) Dynamic stability of planing boats.Mar.tech.,vol.29,no.1, pp.4-12.

[4]Y.Ikeda et al.(2000) Stability of high speed craft.Contemporary Ideas on Ship Stability,D.Vassalos et al.,Eds.New York:Elsevier Science Ltd,pp:401-409.

[5]Prasanta K.Sahoo and Lawrence J. Doctors(2004) Theoretical and Experimental Study of Motion Characteristics of High-Speed Catamaran Hull Forms.9th Symposium on Practical Design of Ships and Other Floating Structures,Luebeck-Travemuende,Germany.

[6]D.Savitsky(1964) Hydrodynamic design of planing hulls.Mar.Tech.,vol.1,no.1,pp:71-95

[7]A.W.Troesch(1992) On the hydrodynamics of vertically oscillating planing hulls.J.ship Res.,vol.36,no.4, pp:317-331

[8] A.W.Troesch and J.M.Falzarano(1993) Modern nonlinear dynamical analysis of vertical plane motion of planing hulls.J.ship Res.,vol.37,no.3, pp:189-199

[9]M.Martin(1978) Theoretical determination of porpoising instability of high speed planing boats. J.ship Res.,vol.22,no.1, pp:32-53

[10] M.Martin(1978) Theoretical prediction of motions of high speed planing boats in waves. J.ship Res.,vol.22,no.3, pp:140-169 
[11]J.D.Hicks,A.W.Troesch,and C.Jiang(1995) Simulation and nonlinear dynamics analysis of planing hulls.J.Offshore Mech.Arctic Eng.,vol.117,pp:38-45

[12]Yumin Su, Qingtong Chen, Hailong Shen, Wei Lu(2012). Numeical simulation of a planing vessel at high speed. J.Marine Sci.Appl. 11:178-183.

[13]Hui Sun, Odd M. Faltinsen(2011). Predictions of porpoising inception for planing vessels. J. Mar. Sci. Technol 16:270:282.

[14]Yao Tie, Shi Yanfeng, Dong Wencai, Yue Guoqiang(2011). Effects of dead rise on the resistance performance of deep-vee planing craft. Ship and Ocean Engneering, vol.40,no.1,pp:29-34

[15]Wang Shuo, Su Yumin, Pang Yongjie,Zhang Xi(2013). Study on the accuracy in the hydrodynamic prediction of high speed planing crafts of CFD method. Journal of Ship Mechanics,vol.17,no.10,pp:1107-1114.

[16]Ma Weijia, Pang Yongjie, Sun Huawei, Yang Zewei(2013). Application of mixed grid in numerical simualtion of planning hull resistance. Ship Engineering,vol.35,no.4,pp:8-10

[17]Sun Huawei, Zou Jin, Huang Debo, Deng Rui(2011). Experimental study on a high speed trimaran planing boat. Journal of Harbin Engineering University,vol.32,no.7,pp:858-861

[18]F. Menter(1992). Influence of freestream values on k-co turbulence model predictions. AIAA.

[19]F. Menter(1994). Two-equation eddy viscosity turbulence models for engineering applications. AIAA Journal, vol. 32, pp. 1299-1310. 